\title{
ESTUDO DA DILUIÇÃO EM JUNTAS SOLDADAS DE AÇO AISI 410S *
}

\section{Resumo}

Beatriz Araújo Batista ${ }^{1}$

Marcela Alves Silva ${ }^{1}$ Geocátia Gonçalves Silva ${ }^{1}$ Reigiane Domingos Marcos ${ }^{1}$ Reginaldo Pinho Barbosa ${ }^{2}$

A soldagem é um processo de união que envolve muitos fenômenos metalúrgicos que podem causar problemas práticos se não forem aplicados os princípios operacionais apropriados ao processo. O aço inoxidável ferrítico AISI 410 S é uma liga de Fe-Cr que contêm em média $12,5 \%$ de cromo, isto melhora a resistência à corrosão, mas em parte sacrifica outras propriedades como a sua soldabilidade. Este estudo foi realizado através da deposição de um cordão de solda em duas chapas de aço AISI 410S pelo processo de eletrodo revestido, variando a energia de soldagem entre eles, onde se calculou a diluição de cada cordão depositado e a composição química da zona fundida de cada chapa. Além disso, foi avaliado através do diagrama de Shaeffler, as microestruturas formadas e os possíveis defeitos susceptíveis mediante os parâmetros utilizados nas chapas. Verificou-se que na chapa onde se aplicou baixa energia de soldagem não ocorreu o risco de defeitos. E na chapa que se aplicou alta energia houve a possibilidade de ocorrer fragilização e fissuração por formação de martensita. Salienta-se que a diluição foi maior nessa condição, mostrando que o controle da diluição em soldas é de grande importância por influenciar na qualidade do cordão de solda.

Palavras-chave: Soldagem; Aço inoxidável ferrítico AISI 410S; Diluição.

\section{Abstract}

\section{DILUTION STUDY ON JOINTS WELDED STEEL AISI $410 S$}

The welding is a process of union involving many metallurgical phenomena that can cause practical problems if they are not applied operational principles appropriate to the process. The ferritic stainless steel AISI $410 \mathrm{~S}$ is an $\mathrm{Fe}-\mathrm{Cr}$ alloy containing on average $12.5 \%$ of chromium, that improves corrosion resistance, but partly sacrificing other properties such as their weldability. This study was carried out by depositing a weld bead on two steel plates AISI 410 S by smaw process by varying the energy of welding between them, which is calculated dilution of each deposited bead and the chemical composition of the molten zone each plate. Furthermore, it was evaluated by Shaeffler diagram, the formed microstructures and possible defects likely due to the parameters used in the plates. It was found that the sheet where welding energy low applied not occur the risk of defects. And the plate was applied high energy welding there is a possibility of embrittlement and cracking formation of martensite. Please note that the dilution was higher in this condition, showing that the control of dilution in solders is of great importance to influence the quality of the weld bead.

Keywords: Welding; ferritic stainless steel AISI 410S; Dilution.

1 Graduanda em Engenharia Metalúrgica, Centro Universitário do Leste de Minas Gerais - Unileste, Coronel Fabriciano, Minas Gerais, Brasil.

2 Engenheiro Mecânico, M.Sc., Professor/Pesquisador de Inox, Centro Universitário do Leste de Minas Gerais/Aperam South America, Cel. Fabriciano/Timóteo, Minas Gerais, Brasil. 


\section{INTRODUÇÃO}

A soldagem é um processo de união de metais e/ou não metais que pode ser realizada através da fusão, pressão, deposição de material fundido, entre outros. Ela envolve muitos fenômenos metalúrgicos como, por exemplo, fusão, solidificação, transformações no estado sólido, deformações causadas pelo calor e tensões de contração e tração, que podem causar muitos problemas práticos se não for aplicado os princípios metalúrgicos apropriados ao processo. A soldagem pode ser dividida em dois grandes grupos de processos: pressão e fusão [1].

A soldagem com Eletrodos Revestidos (Shielded Metal Arc Welding - SMAW) é um processo de soldagem por fusão no qual a coalescência dos metais é obtida pelo aquecimento destes com um arco estabelecido entre um eletrodo revestido e a peça que está sendo soldada [2]. O eletrodo é formado por um núcleo metálico ("alma"), recoberto por uma camada de minerais e/ou outros materiais (revestimento). A alma do eletrodo conduz a corrente elétrica e serve como metal de adição. O revestimento gera escória e gases que protegem da atmosfera a região sendo soldada e estabilizam 0 arco. O revestimento pode ainda conter elementos que são incorporados à solda, influenciando sua composição química e características metalúrgicas [1].

A composição final do metal de solda é o resultado de uma mistura do eletrodo ou do metal de adição fundido com o metal de base que é fundido. O metal depositado do eletrodo ou do metal de adição é denominado "diluído" pelo metal de base fundido. Quando nenhum metal é adicionado, consistindo então o metal de solda inteiramente de metal de base, a diluição é definida como 100\%. Na soldagem manual com eletrodo revestido, o passe de raiz pode ter $30 \%$ de diluição e os passes subsequentes terão uma diluição ligeiramente menor [3].

Como resultado da uniformidade do metal de solda, é possível calcular sua composição se as proporções de metal de base e de eletrodo fundido puderem ser estimadas. Isso pode ser feito frequentemente de uma observação da seção reta da solda, tais cálculos são importantes quando é utilizado um eletrodo ou metal de adição de composição diferente da do metal de base [3].

O aço inoxidável ferrítico AISI 410S é uma liga de Fe-Cr com estrutura cubica de corpo centrado (CCC) que possui cerca de 11,5 a $13,5 \%$ de cromo e menos de $0,08 \%$ de carbono. Deve-se tomar cuidado ao soldar esse tipo de aço para evitar o crescimento de grão e a formação de microestruturas indesejáveis [4].

O objetivo deste trabalho foi à deposição de um cordão de solda em duas chapas de aço inoxidável ferrítico AISI 410 S pelo processo de eletrodo revestido, variando a energia de soldagem entre as chapas e posteriormente, calcular a diluição de cada cordão depositado e a composição química da zona fundida de cada chapa. Além disso, procurou-se verificar através do diagrama de Shaeffler, adaptado por Bystram, as microestruturas formadas e os susceptíveis problemas de ordem metalúrgica na zona fundida que poderão ocorrer ou não a partir dos parâmetros utilizados [5].

\section{MATERIAIS E MÉTODOS}

Foram utilizadas duas chapas do aço inoxidável AISI 410 S laminadas a quente e decapadas fornecidas pela Aperam South America, localizada em Timóteo, Minas Gerais. As chapas foram preparadas, e, o cordão depositado na posição plana. Utilizou-se uma fonte retificadora para soldagem com eletrodo revestido. Foram utilizados dois níveis de energia de soldagem (Eab), sendo que na primeira chapa soldada objetivou-se um valor menor ou igual a $400 \mathrm{~J} / \mathrm{mm}$ e na segunda chapa uma 
energia de soldagem superior a $600 \mathrm{~J} / \mathrm{mm}$. Antes de cada experimento utilizou-se uma "chapa de sacrifício" para o auxílio da calibração da fonte retificadora para alcançar uma solda de qualidade de acordo com o valor da corrente e tensão objetivada para a confecção dos experimentos.

A Tabela 1 informa a composição química estimada do metal base e do eletrodo, dados estes informados pelos fabricantes, Aperam e Esab, e, a Tabela 2 apresenta os parâmetros de soldagem inicialmente estimados. A Figura 1 exibe a foto das duas chapas depois de soldadas.

Tabela 1. Composição química do metal de base e do eletrodo (\% em massa)

\begin{tabular}{ccccccc} 
& $\mathbf{C}$ & $\mathbf{M n}$ & $\mathbf{S i}$ & $\mathbf{C r}$ & $\mathbf{N i}$ & Mo \\
\hline 410S & 0,007 & 0,58 & 0,57 & 11,28 & 0,34 & 0,007 \\
E 308L & 0,030 & 0,80 & 0,80 & 19,60 & 9,90 & 0,000 \\
\hline
\end{tabular}

Tabela 2. Parâmetros de Soldagem

\begin{tabular}{cc}
\hline Posição da Solda & Plana \\
\hline Tipo de solda & Cordão reto, sem oscilar. \\
Direção & Da esquerda para a direita \\
Espessura da Chapa & $6 \mathrm{~mm}$ \\
Eletrodo & $\mathrm{E} 308 \mathrm{~L}$ \\
Corrente do Arco & 60 a $100 \mathrm{~A}$ \\
Tensão do Arco & 16 a 27 volts \\
\hline
\end{tabular}

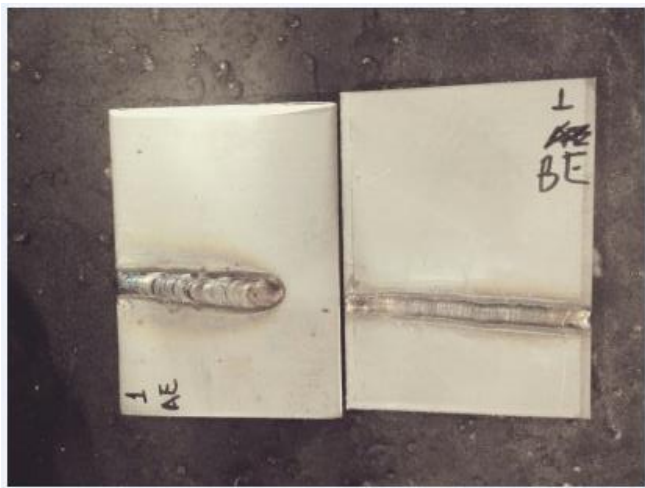

Figura 1. Foto das chapas utilizadas depois de serem soldadas.

A energia de soldagem (Eab) é definida como o calor cedido à junta soldada por unidade de comprimento [3]. As energias de soldagem foram calculadas através da Equação 1.

Onde:

$$
\mathrm{Eab}=\boldsymbol{\eta}_{\mathrm{t}} \frac{\mathbf{V} \mathbf{x} \mathbf{I}}{\mathbf{V s}}
$$

Eab = energia de soldagem $(\mathrm{J} / \mathrm{mm})$

$\boldsymbol{\eta}_{\mathbf{t}}=$ rendimento térmico do processo

V = tensão $(\mathrm{V})$

I = corrente $(A)$

Vs = velocidade de soldagem $(\mathrm{mm} / \mathrm{s})$

O rendimento térmico do material foi considerado de 0,8 de acordo com as literaturas revisadas. As velocidades de soldagem foram determinadas através do comprimento 
dos cordões de soldas, medidos com o auxílio de uma régua milimétrica e o tempo de soldagem determinado através de um cronômetro.

Após a soldagem, removeu-se a escória formada e o resfriamento foi realizado ao ar livre. As chapas foram conduzidas até o Centro de Pesquisa da Aperam South America, onde as mesmas foram preparadas e cortadas nas regiões dos cordões de solda em forma de corpos de prova para a realização de ensaios metalográficos.

A zona fundida é formada por contribuições do metal base e do metal de adição, que são misturados, no estado líquido, na poça de fusão. Define-se como coeficiente de diluição $(\delta)$, ou simplesmente diluição, a proporção com que o metal base participa da zona fundida [1].

A diluição $(\delta)$ pode ser obtida pela medida, em uma macrografia, da seção transversal da solda das áreas proporcionais a quantidade de metal base $(B)$ e de adição $(A)$ fundidos [1], como mostrado na Figura 2 e na Equação 2.

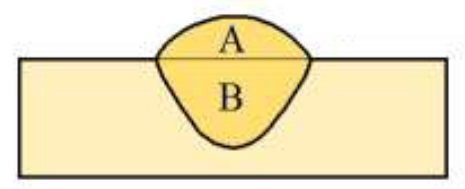

Figura 2. Medida da diluição na seção transversal de um cordão depositado sobre chapa.

$$
\delta=\frac{\mathrm{B}}{\mathrm{A}+\mathrm{B}} \times 100
$$

Através da literatura, decidiu-se utilizar a Equação 3, para calcular a área $A$ corresponde a área do metal de adição e B corresponde a área do metal base diluído das chapas, pois considera-se que estas áreas como iguais a de uma semi elipse.

$$
\mathrm{A}=\frac{\pi * \mathrm{w} * \mathrm{r}}{4}
$$

Com os valores calculados da diluição pode-se estimar a composição química da zona fundida das amostras, a partir da equação 4.

Onde:

$$
\mathrm{CQzf}=\mathrm{CQmb} * \delta+\mathrm{CQma}^{*}(1-\delta)
$$

CQzf = composição química do elemento na zona fundida

$\mathbf{C Q m b}=$ composição química do elemento no metal base

CQma = composição química do elemento no metal de adição

$\boldsymbol{\delta}=$ diluição

O diagrama de Schaeffler permite prever a microestrutura da zona fundida com base na sua composição química e não é restrito aos aços inoxidáveis austeníticos, podendo ser usado também para aços ferríticos e martensíticos. Para utilizá-lo, os equivalentes de $\mathrm{Cr}$ e Ni devem ser calculados pela composição química da solda e a microestrutura é determinada pela leitura direta no diagrama do campo em o ponto (Creq, Nieq) se localiza [6]. Para cálculo do cromo equivalente e níquel equivalente são utilizadas as Equações 5 e 6.

$$
\begin{aligned}
& \text { Creq }=\mathrm{Cr}+\mathrm{Mo}+1,5 * \mathrm{Si}+0,5{ }^{*} \mathrm{Nb} \\
& \text { Nieq }=\mathrm{Ni}+30 * \mathrm{C}+0,5 * \mathrm{Mn}+30{ }^{*} \mathrm{~N}
\end{aligned}
$$


Em aplicações em que as composições dos metais base e de adição sejam diferentes, o ponto que representa a solda no diagrama estará sobre o segmento de reta entre o metal base e o metal de adição. A posição desse ponto no segmento dependerá da diluição da solda, ficando mais próximo do metal de adição para soldas de pequena diluição [6]. A Figura 3 mostra o diagrama de Shaeffler modificado por Bystram para determinação das regiões susceptíveis a problemas de ordem metalúrgica na zona fundida [5].

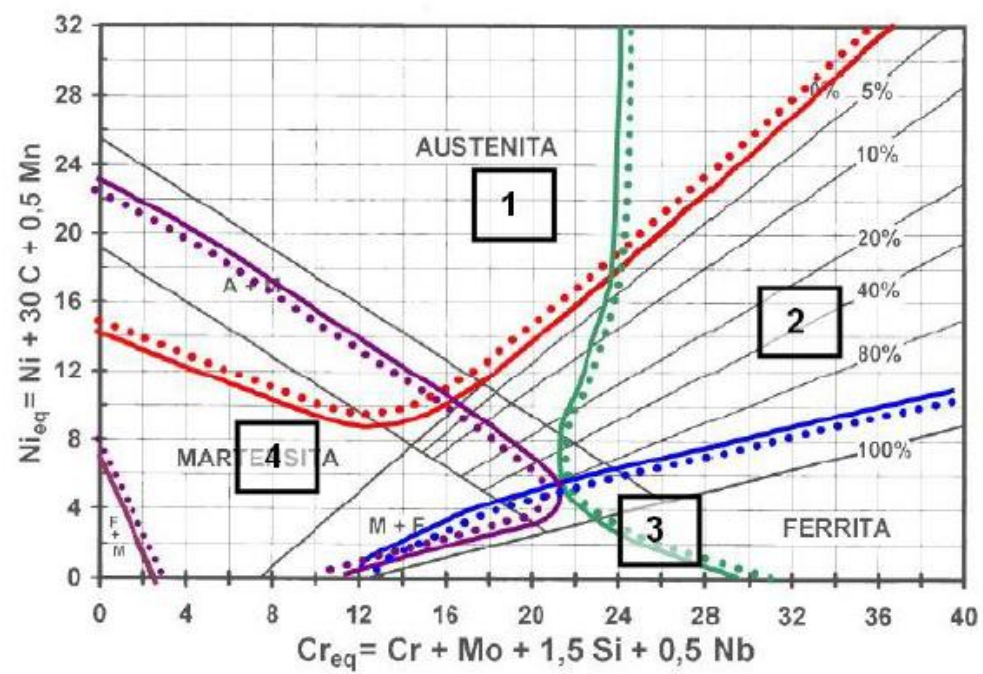

Figura 3. Regiões problemáticas típicas na soldagem de aços inoxidáveis: (1) Formação de trincas de solidificação ou por perda de ductilidade acima de $1250^{\circ} \mathrm{C}$; (2) Fragilização por formação de fases intermetálicas após aquecimento entre cerca de $450^{\circ} \mathrm{C}$ a $900^{\circ} \mathrm{C}$; (3) Fragilização por crescimento de grão; (4) Fragilização e fissuração por formação de martensita.

Lembrando que as composições químicas das soldas que caírem dentro desses campos pode apresentar outros defeitos, mas os principais são os indicados. Assim ao executar a solda, deve-se levar em conta primeiramente a prevenção contra os problemas indicados.

\section{RESULTADOS E DISCUSSÃO}

Para melhor análise dos resultados e discussões decidiu-se avaliar separadamente cada solda em função da intensidade de energia depositada nas mesmas.

\subsection{Baixa energia}

A Tabela 3 mostra os parâmetros obtidos durante a execução da soldagem de baixa energia, onde se objetivava uma energia de soldagem menor ou igual a $400 \mathrm{~J} / \mathrm{mm}$. 
Tabela 3. Dados obtidos durante o processo de soldagem com baixa energia

\begin{tabular}{cc}
\hline & Baixa Energia \\
\hline Tensão (V) & 23,4 \\
Corrente (A) & 52,0 \\
Tempo (s) & 32,0 \\
Comprimento (mm) & 96,0 \\
Energia de soldagem - E1 (J/mm) & 324,5 \\
Velocidade de soldagem (mm/s) & 3,0 \\
Tensão da fonte em vazio (V) & 58,3 \\
Rendimento térmico - Nt & $80 \%$ \\
\hline
\end{tabular}

A partir da macrografia mostrada na Figura 4 pode-se calcular as dimensões do cordão de solda e utilizando-se a Equação 3. Foram calculadas as área $A 1$ corresponde a área do metal de adição e a área B1 corresponde a área do metal base diluído.

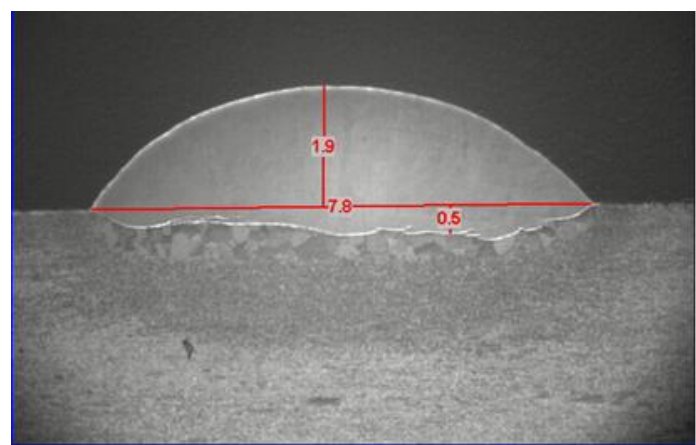

Figura 4. Dimensões do cordão de solda com baixa energia de soldagem.

- Cálculo da área do metal de adição (A1):

$\mathrm{A} 1=\frac{\pi * 7,8 * 1,9}{4}=11,64 \mu \mathrm{m}$

- Cálculo da área diluida do metal base (B1):

$\mathrm{B} 1=\frac{\pi * 7,8 * 0,5}{4}=3,06 \mu \mathrm{m}$

Com as áreas calculadas e a Equação 2 pode-se calcular a diluição. 0 resultado obtido foi:

$\delta=\frac{3,06}{3,06+11,64} \times 100=21 \%$

Utilizando-se a Equação 4 calculou-se a composição química da zona fundida e pelas Equações 5 e 6 o cromo equivalente e o níquel equivalente, respectivamente. Os resultados obtidos se encontram na Tabela 4:

Tabela 4. Composição da zona fundida - baixa energia (\% em massa)

\begin{tabular}{|c|c|c|c|c|c|c|c|}
\hline $\mathbf{C}$ & $\mathbf{M n}$ & $\mathbf{S i}$ & $\mathbf{C r}$ & $\mathbf{N i}$ & Mo & Creq & Nieq \\
\hline $0,025 \%$ & $0,754 \%$ & $0,751 \%$ & $17,850 \%$ & $7,890 \%$ & $0,002 \%$ & $18,98 \%$ & $9,02 \%$ \\
\hline
\end{tabular}


Com os valores calculados de cromo e níquel equivalentes e utilizando o diagrama de Shaeffler adaptado por Bystram (Figura 3), verificou-se que a microestrutura do cordão de solda constitui-se de $13 \%$ de ferríta e $87 \%$ de austenita (Figura 5) e está localizado em uma região não susceptível à ocorrência de defeitos de ordem metalúrgica.

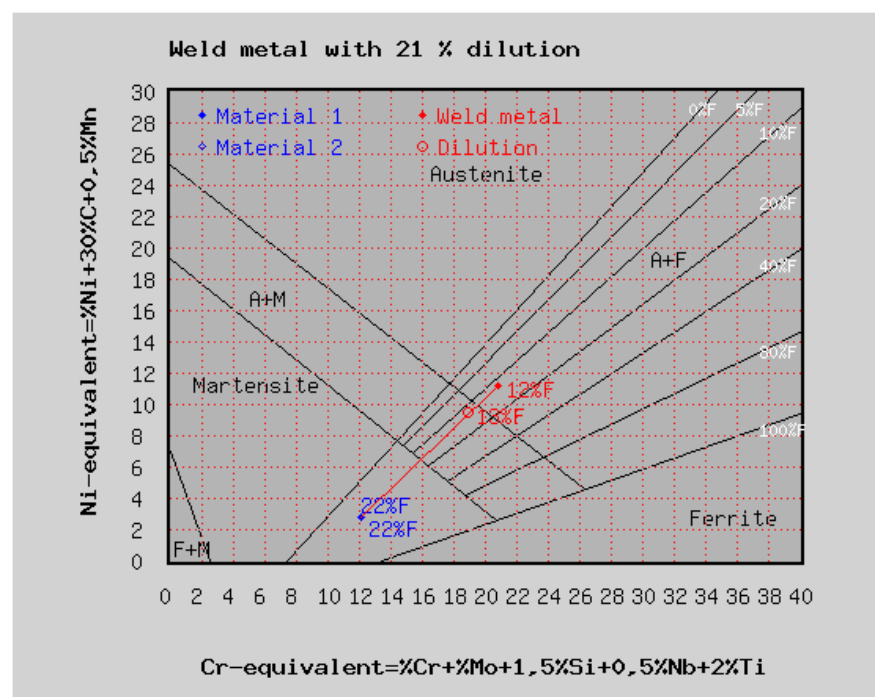

Figura 5. Resultado da microestrutura do cordão de solda com baixa energia de soldagem.

\subsection{Alta Energia}

A Tabela 5 mostra os parâmetros obtidos durante a execução da soldagem de alta energia, onde se objetivava uma energia de soldagem superior a $600 \mathrm{~J} / \mathrm{mm}$.

Tabela 5: Dados obtidos durante o processo de soldagem com alta energia

\begin{tabular}{cc}
\hline \multicolumn{1}{c}{ Alta Energia - B } \\
\hline Tensão (V) & 26,0 \\
Corrente (A) & 64,0 \\
Tempo (s) & 35,0 \\
Comprimento (mm) & 67,0 \\
Energia de soldagem - E2 (J/mm) & 696,9 \\
Velocidade de soldagem (mm/s) & 1,9 \\
Tensão da fonte em vazio (V) & 60,3 \\
Rendimento térmico - Nt & $80 \%$ \\
\hline
\end{tabular}

A partir da metalografia mostrada na Figura 6 pode-se calcular as dimensões do cordão de solda e utilizando-se a Equação 6, foram calculadas as área A2 corresponde a área do metal de adição, B2 e B3 correspondes a área diluída do metal base. 


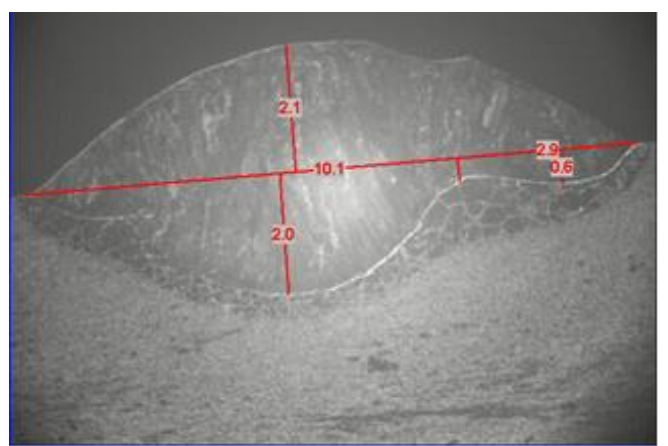

Figura 6. Dimensões do cordão de solda com alta energia de soldagem.

- Cálculo da área do metal de adição (A2):

$\mathrm{A} 2=\frac{\pi * 10,1 * 2,1}{4}=16,66 \mu \mathrm{m}$

- Cálculo da área do metal base (B2 + B3):

$\mathrm{B} 2=\frac{\pi * 2,9 * 0,6}{4}=1,37 \mu \mathrm{m}$

$\mathrm{B} 3=\frac{\pi * 7,2 * 2}{4}=11,31 \mu \mathrm{m}$

$\mathrm{B} 2+\mathrm{B} 3=1,37+11,31=12,68 \mu \mathrm{m}$

Com as áreas calculadas e a equação 2 pode-se calcular a diluição. O resultado obtido foi:

$\delta=\frac{12,68}{12,68+16,66} \times 100=43 \%$

Utilizando-se a Equação 4 calculou-se a composição química da zona fundida e pelas Equações 5 e 6 o cromo equivalente e o níquel equivalente, respectivamente. Os resultados obtidos se encontram na Tabela 5.

Tabela 5. Composição da zona fundida alta energia (\% em massa)

\begin{tabular}{|c|c|c|c|c|c|c|c|}
\hline $\mathbf{C}$ & $\mathbf{M n}$ & $\mathbf{S i}$ & $\mathbf{C r}$ & $\mathbf{N i}$ & Mo & Creq & Nieq \\
\hline $0,020 \%$ & $0,705 \%$ & $0,701 \%$ & $16,020 \%$ & $7,790 \%$ & $0,003 \%$ & $17,07 \%$ & $6,74 \%$ \\
\hline
\end{tabular}

Com os valores calculados de cromo e níquel equivalentes e utilizando o diagrama de Shaeffler adaptado por Bystram (Figura 4), verificou-se que a microestrutura do cordão de solda constitui-se de $15 \%$ de ferríta e $85 \%$ de austenita (Figura 7 ) e está localizado em uma região propensa à ocorrência de defeitos como fragilização e fissuração por formação de martensita. Este fato pode ser explicado pelo maior valor da diluição, implicando numa participação maior do metal base ferrítico na formação da zona fundida. 
Weld metal with $43 \%$ dilution

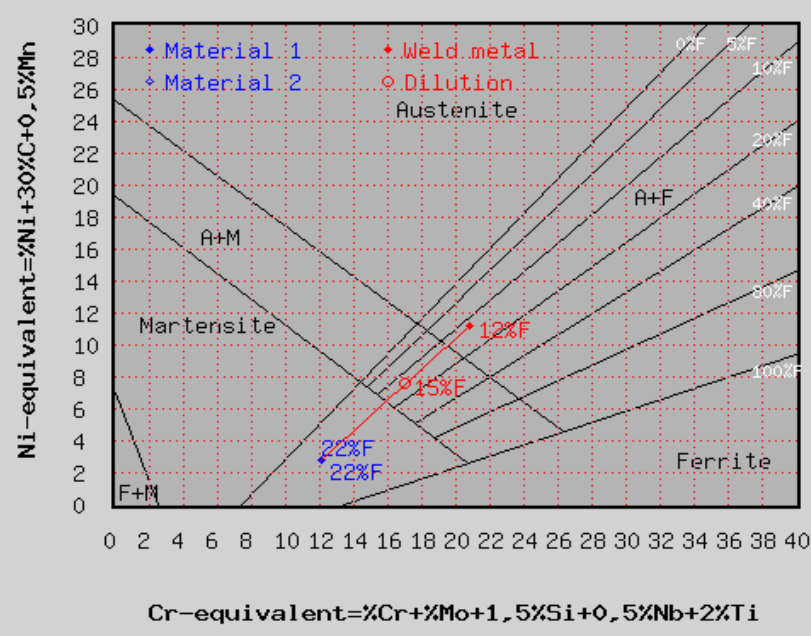

Figura 7. Resultado da microestrutura do cordão de solda com alta energia de soldagem.

\section{CONCLUSÃO}

Através do estudo realizado pode-se concluir que:

- É possível calcular a diluição de soldas de cordão depositado sobre chapas;

- Maior energia de soldagem leva a maiores valores de diluição, o que por vezes pode ser prejudicial ao resultado final da soldagem;

- A partir do cálculo da diluição torna-se possível calcular a composição química da zona fundida;

- A diluição mais elevada pode tornar-se indesejável em soldas de aços inoxidáveis ferríticos AISI 410S devido à possibilidade de tornar a zona fundida susceptível à fragilização e fissuração por formação de martensita;

- O aumento da diluição modificou a participação das fases e a composição química das zonas fundidas, mesmo sendo pequena a diferença, tal circunstância fez com que a solda de alta energia ficasse localizada em uma zona do diagrama de Shaeffler propensa a defeitos;

- De forma geral, conclui-se que o controle da diluição em soldas é de grande importância tendo em vista que a mesma pode influenciar a qualidade final do cordão de solda.

\section{REFERÊNCIAS}

1 MODENESI, P. J. MARQUES, P. V. DAGOBERTO, B. S. Introdução à Metalurgia da Soldagem. Apostila. Universidade Federal de Minas Gerais. Belo Horizonte, MG. 2012.

2 MARQUES, P.V.; MODENESI, P.J.; BRACARENSE, A. Q. Soldagem: Fundamentos e Tecnologia. 2.ed. Minas Gerais: UFMG, 2007. 362p.

3 FORTES, C. Metalurgia da Soldagem. Apostila ESAB BR. 2004.

4 CARBO, H. M. Aço Inoxidável: Aplicações e Especificação. Apostila Núcleo Inox. Timóteo. 2001.

5 DRAPINSKI, J. Elementos de Soldagem: Manual Prático de Oficina. São Paulo: Mc Graw-Hill do Brasil, LTDA. 1979.

6 Denver soldas. Diagrama de Schaeffler. Disponível em: <http://www.denversa.com.br/ site/files/produto/b6fb82c75c9074945a16bb90250a8ba0.pdf>. Acesso em: 18 de Janeiro de 2016. 